\title{
Immunity to SARS-CoV-2 in a Dialyzed Patient Who Developed COVID-19 Twenty Days After the Second Dose of BNT162b2 Vaccine: a Case Report
}

\section{Sabrina MANNI ( $\sim$ s.manni@ch-cannes.fr)}

CH Cannes: Centre Hospitalier de Cannes https://orcid.org/0000-0002-2916-6771

\section{Laurène LOTTE}

CH Cannes: Centre Hospitalier de Cannes

Antonin BAL

Hospices Civils de Lyon

Laurence JOSSET

Hospices Civils de Lyon

Bruno LINA

Hospices Civils de Lyon

Mary-Anne TRABAUD

Hospices Civils de Lyon

Gregory DESTRAS

Hospices Civils de Lyon

Bruno POZZETTO

CHU Saint-Étienne: Centre Hospitalier Universitaire de Saint-Etienne

Martine VALETTE

Hospices Civils de Lyon

Corinne PASSERON

CH Cannes: Centre Hospitalier de Cannes

Barbara SEITZ-POSLKI

CHU Nice: Centre Hospitalier Universitaire de Nice

Audrey SINDT

CH Cannes: Centre Hospitalier de Cannes

Matteo VASSALLO

CH Cannes: Centre Hospitalier de Cannes

\section{Research Article}

Keywords: SARS-CoV-2, COVID-19 vaccine, dialyzed patient, End-stage kidney disease, BNT162b2, immune response 
Posted Date: July 21st, 2021

DOI: https://doi.org/10.21203/rs.3.rs-711429/v1

License: (c) (i) This work is licensed under a Creative Commons Attribution 4.0 International License. Read Full License 


\section{Abstract}

Introduction

End stage kidney disease (ESKD) and cancer have been identified as risk factors for severe and fatal cases of COVID-19, making vaccination in these patients a priority. Patients suffering from ESKD have a significantly weaker response to common vaccines than general population. However, humoral and cellular immune responses after two doses of RNA-based vaccine BNT162b2 (Pfizer-BioNTech) have been poorly explored in this vulnerable population.

Case presentation

A 69-year-old male patient was followed for ESKD and myeloma. He developed a severe SARS-CoV-2 pneumonia twenty days after two doses of BNT162b2 vaccine. Whole genome sequencing found that the virus belonged to the 20l/501Y.V1 clade. A serology draws eight days after the $2^{\text {nd }}$ vaccine dose showed positive RBD IgG without neutralizing activity. A serum specimen sampled thirty days after the onset of SARS-CoV-2 infection showed seroconversion against both RBD and $\mathrm{N}$ antigens. This specimen was shown to exhibit a frank neutralizing activity. The QuantiFERON® SARS-CoV-2 (Qiagen) showed a positive specific cellular response although the QuantiFERON monitor displayed a weak cellular response.

Conclusions

Impaired immunity due to renal failure probably explain the severe pneumonia despite vaccination. The fact that the patient developpe a neutralizing activity and a cellular response after a third stimulation by infection may suggest to systemically administrate a third dose of vaccine in ESKD patients.

\section{Introduction}

End stage kidney disease (ESKD) and cancer have been identified as risk factors for severe and fatal cases of COVID-19[1], making vaccination in these patients a priority. Patients suffering from ESKD have a significantly weaker response to common vaccines than general population [2]. However, humoral and cellular immune responses after two doses of RNA-based vaccine BNT162b2 (Pfizer-BioNTech) have been poorly explored in this vulnerable population.

\section{Case Presentation}

A 69-year-old male patient was followed for ESKD and myeloma treated with Daratumumab. He received his first injection of BNT162b2 vaccine on January 2021, 20th and the second one 21 days after, on February 10th. Twenty days after the second dose (March, 1st), the patient was hospitalized at Cannes hospital for fever, asthenia, and polypnea. The patient was tested positive for SARS-CoV-2 on nasopharyngeal sample by a RT-PCR assay performed with the NeuMoDX® (Qiagen) instrument cycle threshold values for targeted genes (N and NSP2) were 13.1 and 14.4, respectively. Whole genome 
sequencing found that the virus belonged to the 20I/501Y.V1 clade (GISAID number: EPI_ISL_1312948) with no additional mutation in the spike gene. The blood cell count showed lymphopenia $(0.34 \mathrm{G} / \mathrm{L}$, normal range $1-4 \mathrm{G} / \mathrm{L}$ ) but no inflammatory syndrome (C-Reactive Protein $<20 \mathrm{mg} / \mathrm{L}$ ). The patient developed fever for 8 days and exhibited an oxygen saturation $<93 \%$. Computed tomography of the chest showed bilateral ground-glass opacities involving 25 to $50 \%$ of the lung area. The patient was treated with oxygen supplementation $(3 \mathrm{~L} / \mathrm{min})$ and dexamethasone for 8 days with a favorable outcome. $A$ serological test performed before vaccination showed neither receptor binding domain (RBD) (Atellica ${ }^{\circledR}$, Siemens) nor antigen $\mathrm{N}$ specific antibodies (Architect ${ }^{\circledR}$, Abbott) to SARS-CoV-2. The second serum specimen sampled eight days after the 2nd vaccine dose showed positive RBD IgG [3.92 arbitrary units $(\mathrm{AU}) / \mathrm{mL}$, positive threshold $>1 \mathrm{AU} / \mathrm{mL}$ ] and negative $\mathrm{N} / \mathrm{gG}$. A third serum specimen sampled thirty days after the onset of SARS-CoV-2 infection showed a clear seroconversion against both RBD (IgG > 150 $\mathrm{AU} / \mathrm{mL}$ ) and $\mathrm{N}$ antigens (IgG index of 7.53) (Fig. 1). Despite a weak RBD humoral response after vaccination, the patient developed SARS-CoV-2 pneumonia, which raises the question of vaccine efficacy. To better evaluate the humoral protection against SARS-CoV-2, an in vitro virus neutralization assay, using three live viral isolates, was performed on the three serum samples as previously described[3]. Whereas no neutralizing activity was observed on the first two serum specimens (titer $<20$ ), including the post-vaccination specimen that exhibited a low level of RBD antibodies, the post-infection specimen was shown to exhibit a frank neutralizing activity with an higher titer against the 20I/501Y.V1 virus (titer of 640) than against the reference virus (titer of 240) or the 20H/501Y.V2 virus (titer of 80) (Fig. 1). This observation may explain COVID-19 infection in this patient despite complete vaccination.

Impaired humoral responses are well-known in patients on chronic dialysis against vaccines as hepatitis $B$ and influenza [4], which may presume a poor answer against SARS-CoV-2 vaccination. Canas et al. [5] also showed a lack of correlation between neutralizing activity and conventional serology testing on convalescent serum specimens from pediatric dialyzed patients. These observations raise the question of straightening SARS-CoV-2 immunization in dialyzed patients, as recommended with double-dose hepatitis $B$ vaccination in this population[4]. On the sample collected after the infection, interferongamma release assay (IGRA) by QuantiFERON Monitor showed a low IFN-y level $(3.32 \mathrm{UI} / \mathrm{ml})$ while the QuantiFERON® SARS-CoV-2 (Qiagen) showed a positive specific cellular response. A limit of this case report results is the absence of cellular investigation on the serum specimen taken after the second dose of vaccine to compare cellular immune activity before and after the SARS-CoV-2 pneumonia. Interestingly, the natural infection of this patient with the 20l/501Y.V1 virus boosted both his protective humoral and cellular immune responses to SARS-CoV-2. Another original feature of this case report is the concomitant treatment of this patient with Daratumumab for multiple myeloma. This treatment cannot be made responsible for the poor immune response to SARS-CoV-2 vaccine since Daratumumab was shown to expand both the helper and cytotoxic T-cell repertoires [6].

The chronology of events reported herein needs also to be emphasized; indeed, the SARS-CoV-2 infection occurred within 20 days after the second dose of vaccine, as recently reported by Hacisuleyman et al. in two women infected with SARS-CoV-2 variants despite successful vaccination by BNT162b2 [7]. Unlike these two women our patient presented a severe infection probably explained by impaired humoral 
immunity due to renal failure. This may suggest to systemically administrate a third dose of vaccine in ESKD patients. Prospective studies are necessary to evaluate the efficiency of this scheme in ESKD patients.

\section{Declarations}

\section{Acknowledgements}

The authors acknowledge the staff of the Cannes hospital laboratory for the realization of SARS-CoV-2 PCR.

\section{Funding}

Not applicable.

\section{Competing interests}

The authors declare they have no conflict of interest.

\section{Ethics approval and consent to participate}

Informed consent has been obtained from the patient through an information letter.

\section{Consent for publication}

Written informed consent was obtained from the patient for the publication of this Case Report.

\section{Availability of data and materials}

Not applicable.

\section{Code availability}

Not applicable.

\section{Authors' contributions}

SM and LL wrote the manuscript. All the authors have reviewed the case report and approved the final version.

\section{References}

1. Ozturk S, Turgutalp K, Arici M, Odabas AR, Altiparmak MR, Aydin Z, et al. Mortality analysis of COVID19 infection in chronic kidney disease, haemodialysis and renal transplant patients compared with 
patients without kidney disease: a nationwide analysis from Turkey. Nephrol Dial Transplant Off Publ Eur Dial Transpl Assoc - Eur Ren Assoc 2020;35:2083-95. https://doi.org/10.1093/ndt/gfaa271.

2. Boyer-Suavet S, Cremoni M, Dupeyrat T, Zorzi K, Brglez V, Benzaken S, et al. Functional immune assay using interferon-gamma could predict infectious events in end-stage kidney disease. Clin Chim Acta 2020;502:287-92. https://doi.org/10.1016/j.cca.2019.11.018.

3. Bal A, Pozzetto B, Trabaud M-A, Escuret V, Rabilloud M, Langlois-Jacques C, et al. Evaluation of HighThroughput SARS-CoV-2 Serological Assays in a Longitudinal Cohort of Patients with Mild COVID19: Clinical Sensitivity, Specificity, and Association with Virus Neutralization Test. Clin Chem 2021;67:742-52. https://doi.org/10.1093/clinchem/hvaa336.

4. Centers for Disease Control. Guidelines for vaccinating kidney dialysis patients and patients with chronic kidney disease. Summary of ACIP recommandation 2012.

5. Canas JJ, Starr MC, Hooks J, Arregui S, Wilson AC, Carroll AE, et al. Longitudinal SARS-CoV-2 seroconversion and functional heterogeneity in a pediatric dialysis unit. Kidney Int 2021;99:484-6. https://doi.org/10.1016/j.kint.2020.11.014.

6. Krejcik J, Casneuf T, Nijhof IS, Verbist B, Bald J, Plesner T, et al. Daratumumab depletes CD38+ immune regulatory cells, promotes T-cell expansion, and skews T-cell repertoire in multiple myeloma. Blood 2016;128:384-94. https://doi.org/10.1182/blood-2015-12-687749.

7. Hacisuleyman E, Hale C, Saito Y, Blachere NE, Bergh M, Conlon EG, et al. Vaccine Breakthrough Infections with SARS-CoV-2 Variants. N Engl J Med 2021;0:null. https://doi.org/10.1056/NEJMoa2105000.

\section{Figures}




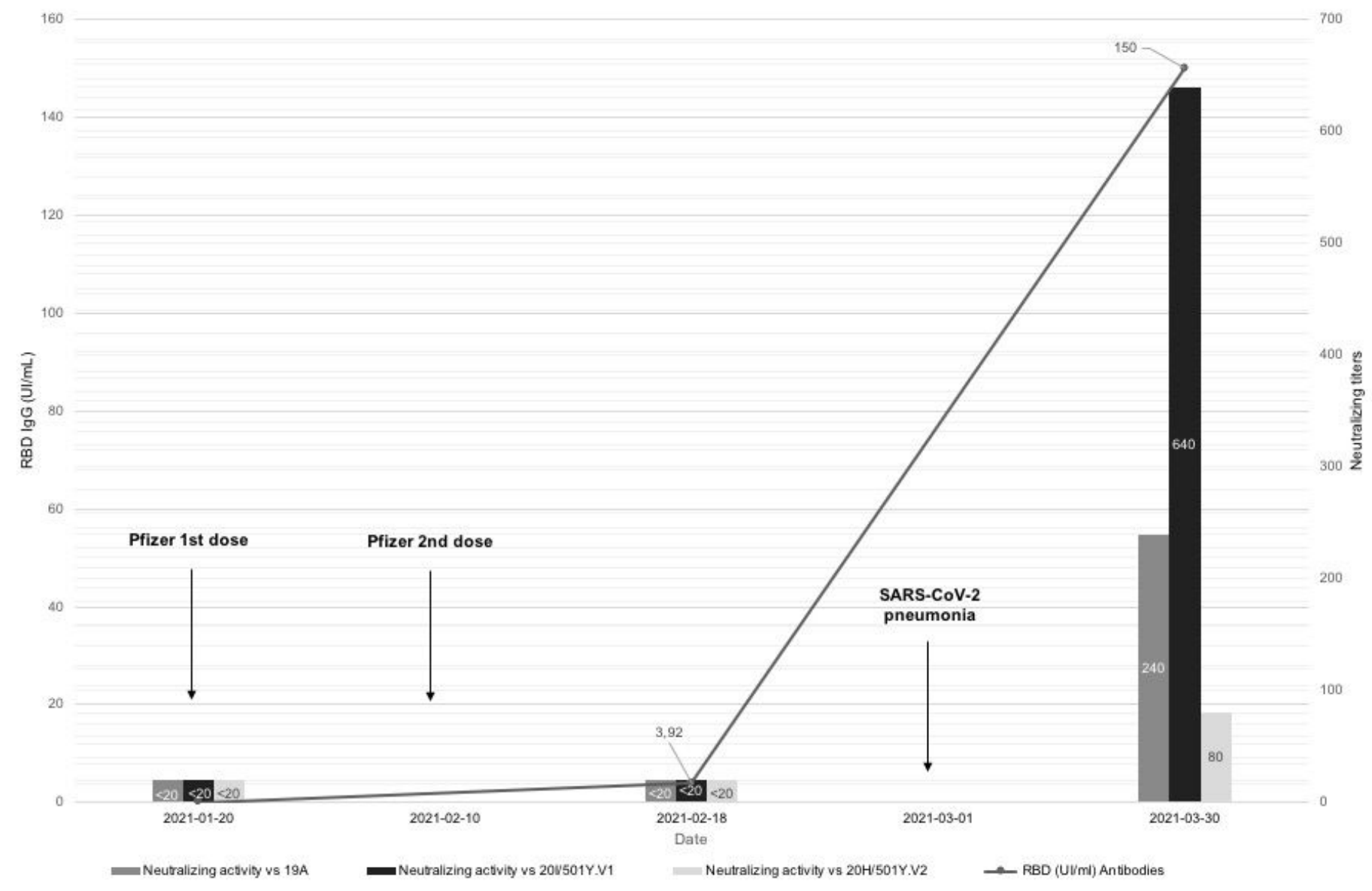

Figure 1

Antibody dynamics to SARS-CoV-2 in a vaccine breakthrough case

\section{Supplementary Files}

This is a list of supplementary files associated with this preprint. Click to download.

- CamScanner0712202117.41.pdf 